\title{
CONGENITAL LAXITY OF THE LIGAMENTS WITH HYPOTONIA
}

\author{
BY \\ PINCUS CATZEL \\ From the Belgrave Hospital for Children, London \\ (RECEIVED FOR PUBLICATION JANUARY 16, 1955)
}

Extreme mobility of the joints is of great advantage to an acrobat, permitting him to assume fascinating postures. When the condition appears in infancy, however, it may be quite alarming, as it may easily be confused with serious organic disease if the features are not known.

There are two basic disturbances in this condition: (1) Abnormal mobility of the bones at the joints, and (2) extreme hypotonia of the muscles. In describing their cases, orthopaedic surgeons have tended to emphasize the former aspects, while physicians have tended to emphasize the latter.

The majority of recorded cases have appeared in the American literature, the first author to draw attention to this anomaly in infancy being Finkelstein, who in 1916 described a 21-month-old child with extreme mobility of all the joints and marked hypotonia. He carefully distinguished the condition from Oppenheim's disease by the fact that there was no gross neurological disturbance in his case, the reflexes were normal and the electrical reactions of the muscles were normal. He called the condition 'joint hypotonia'-an obvious misnomer. Sobel (1926) suggested the term 'essential or primary hypotonia', while Key (1927), Michaels and Searle

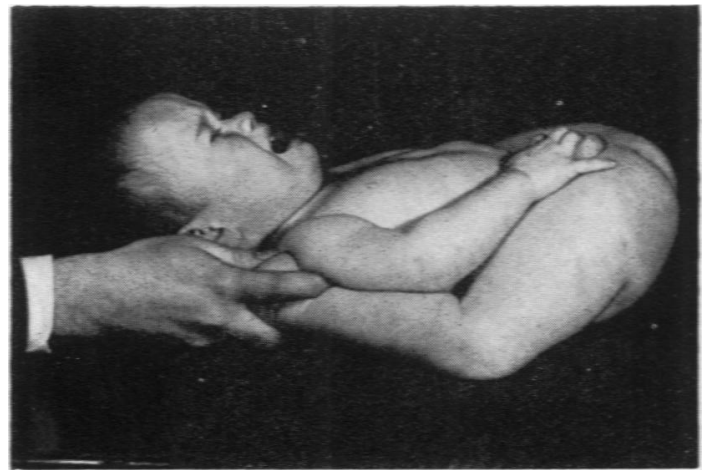

Fig. 1.-Photograph showing extreme mobility of the hip joint. The patient is not in pain, despite the expression on his face. He was hungry and tired.
(1933) and Sturkie (1941) emphasized the 'hypermobility of the joints'. Sutro (1947), on the other hand, described his cases under the title 'Hypermobility of Bone due to "Overlengthened" Capsular and Ligamentous Tissue', thus implying that the anatomical basis of the condition lay primarily in the joint structure rather than in the hypotonic 'overlengthened' muscle and tendon. Lidge (1954) recently described a case under the simple title of 'Hypotonia', while Ford (1952) called it 'Congenital Laxity of the Ligaments (Congenital Atonia)'. For practical purposes I think that "congenital laxity of the ligaments with hypotonia' covers these cases adequately.

\section{Case Report}

I.S. (No. 25925), a boy, was born on December 10, 1953. The patient was referred to the Out-patient Clinic of the Belgrave Hospital for Children by Dr. J. A. Gavin, to whom I am indebted for many of the details of the patient's early history.

The infant was delivered at home and was a full-term, normal delivery, birth weight being $7 \mathrm{lb}$. He had a strong cry and was of good colour. However, it was noted that he was unusually flabby and tended to slip

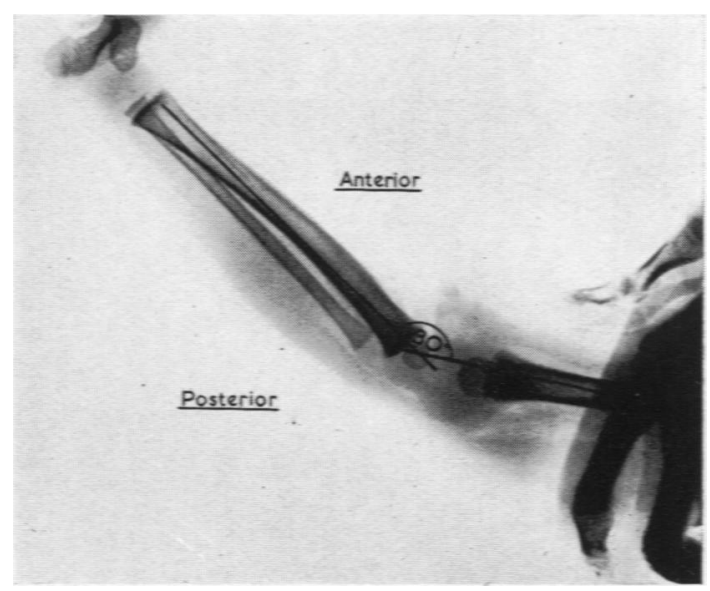

Fig. 2.-Hyperextension at knee-joint through an angle of $30^{\circ}$ 


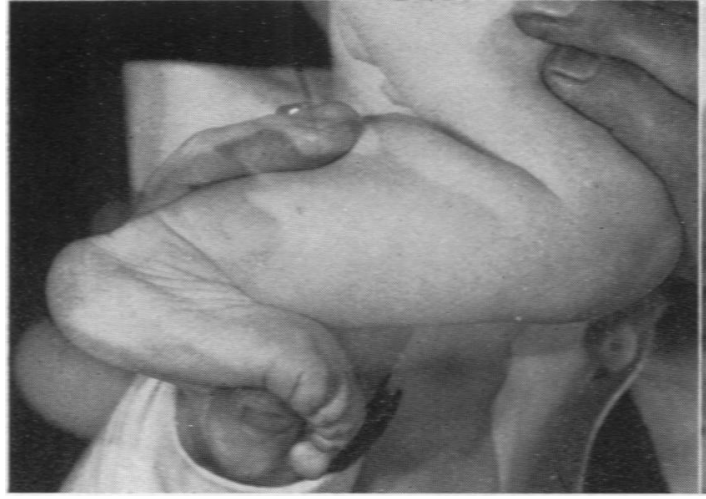

FKG. 3.-Marked dorsifiexion of ankle.

through the fingers when lifted up under the armpits. He tended to lie very passively but fed well. He was breast fed for nine months and fully weaned by 10 months of age. He was an only child and it was found that a nephew of his father had a similar condition. Neither ố the parents had seen this child, as he lived a considerable distance away.

At the age of 4 months the child was still unable to lift his head and tended to lie very passively on his back. The movements of his eyes showed that he took an active interest in his surroundings. His forehead appeared to be bossed and as both fontanelles were widely patent it was thought that he might have rickets. Large doses of vitamin $\mathbf{D}$ were given without effect.

The child was seen for the first time at this hospital at the age of 10 months because of a possible neurological disorder or resistant rickets.

On examination he was found to be a bright-eyed, cooperative, well nourished child who lay passively on the couch and made few movements. The forehead was rounded but there were no stigmata of rickets, mongolism or cretinism. The anterior fontanelle was large and the posterior fontanelle was closed. The skin was healthy. Palpation of the muscles gave the impression that there

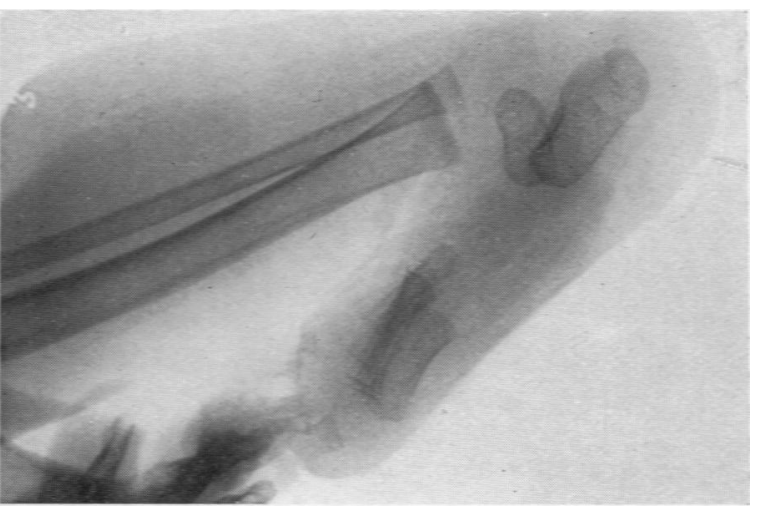

FKG. 3A.-Extreme dorsiflexion of ankle.

were no muscle masses at all and that one was palpating fatty tissue only. The abdomen was protuberant.

The patient could sit for a short time with support, but tired rapidly and would slump. There was an abnormal range of movements at all the joints, some of the abnormal postures being illustrated in the radiographs and photographs. Fig. 1 shows the extreme mobility at the hip joint, the movement being painless. Hyperextension of the knee is seen in Fig. 2. Figs. 3 and 4 show the extremes of dorsi- and plantar-flexion at the ankle joint. This mobility is well seen in the radiographs (Figs. 3A and 4A). A photograph of the wrist in full fiexion and a radiograph in full extension are also shown (Figs. 5 and 5A). Note particularly the angulation at the metacarpo-phalangeal joint. The absence of pain on extreme angulation of the joints was striking. On lifting the child from the supine position with the examining forearm in the hollow of the lumbar spine, he would double backwards in opisthotonos and made weak attempts to flex the head, knees and hips. The extreme laxity of the joints was most marked at the ankles and wrists which, when passively shaken, behaved like flail joints. Nevertheless, active movements were possible and the child could grasp objects with his hands.

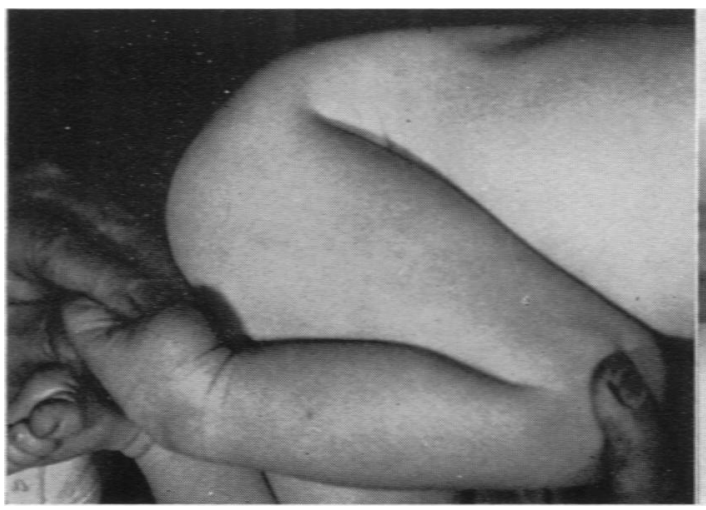

Fig. 4.-Marked plantar flexion of foot.

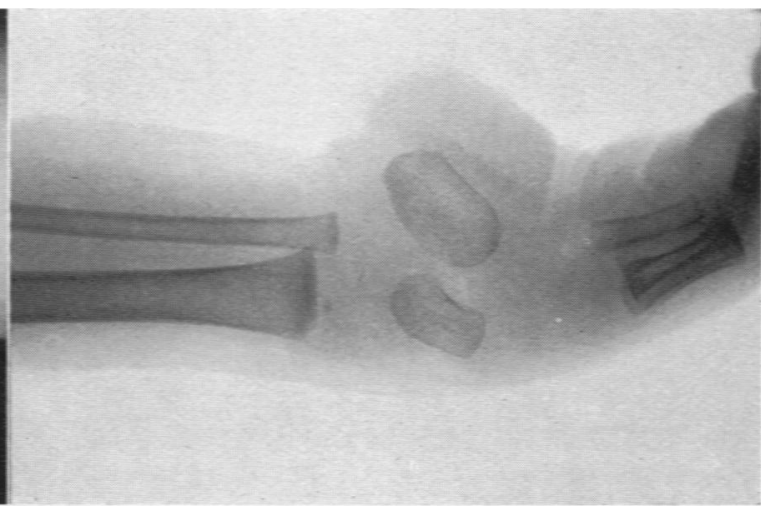

Fig. 4A.-Marked plantar flexion of foot. 


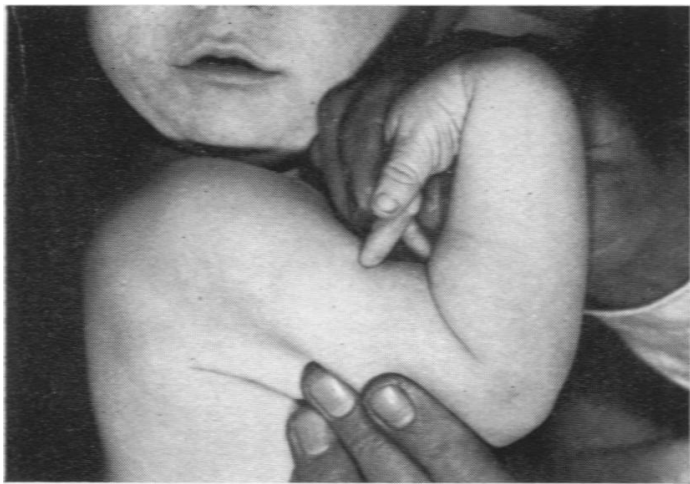

Fig. 5.-Marked flexion at wrist.

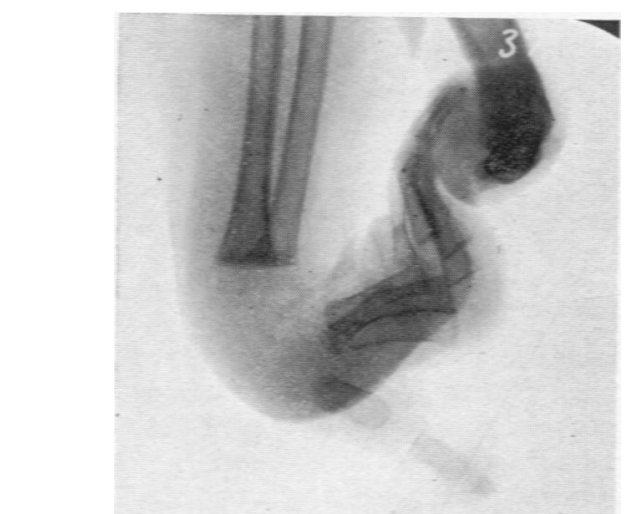

FKG. 5A.-Hyperextension of wrist and metacarpophalangeal joints.

No neurological disorder could be detected, the cranial nerves were intact and the reflexes were all present and normal.

No subluxations were detectable either clinically or radiologically.

Soft-tissue radiographs of the calf muscles showed a normal muscle mass, a useful differentiating feature from amyotonia congenita and Werdnig-Hoffman disease in which atrophy is seen (Figs. 6 and 7).

An electro-diagnostic report from Dr. P. Bauwens, of St. Thomas's Hospital, is as follows:

\begin{abstract}
'Stimulation of the main nerve trunks appeared to produce normal reactions in the muscles supplied by the nerves. Electromyographic exploration of the right tibialis anticus did not reveal any spontaneous activity, while on action, provoked by stroking the sole of the foot, a normal complete interference pattern of motor unit origin was noted.'
\end{abstract}

The patient was seen again two months later at the age of 1 year and showed a remarkable improvement in activity. The mother complained that he had become 'too active' so that it was practically impossible to dress him. He would twist and squirm and was hardly still for a minute. He could pull himself to an upright position, but, owing to the laxity of the ankle joint, could not take steps. On standing, the foot would assume either an everted or inverted position, and, despite its awkwardness, the position was completely painless.

\section{Discussion}

The aetiology of congenital laxity of the ligaments is unknown. The condition is probably congenital, and Finkelstein noted in his case that a grandmother. two uncles, and the mother of the child had had flail joints in infancy which improved in adolescence. In Jahss's (1919) case the paternal grandfather and an uncle had had similar trouble in infancy. Sobel (1926), who has collected the largest series in the literature, noted that other members of the family were affected in four cases. Key (1927) described a family with hypermobile joints involving the father and four sons, whereas the five daughters were normal. It is of interest, however, that all four sons had club-feet in addition, the only joints in the body that were not hypermobile. Key thought that this family illustrated an example of a sex-linked hereditary characteristic. However, Sturkie (1941) reported a family in which all the offspring in two generations (nine cases in all) had hypermobility of the joints to greater or lesser degree. Of these, three

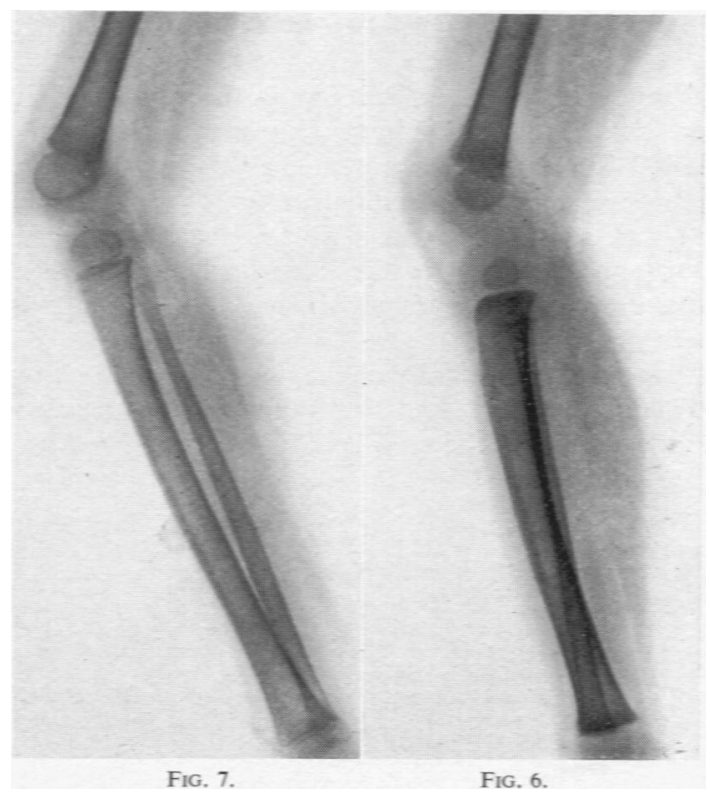

FiG. 6.-Soft tissue radiograph to show normal muscle mass. Fig. 7.-Case of Werdnig-Hoffman disease to show atrophy of muscle mass. 
TABLE 1

SUMMARY OF CASES REPORTED IN THE LITERATURE

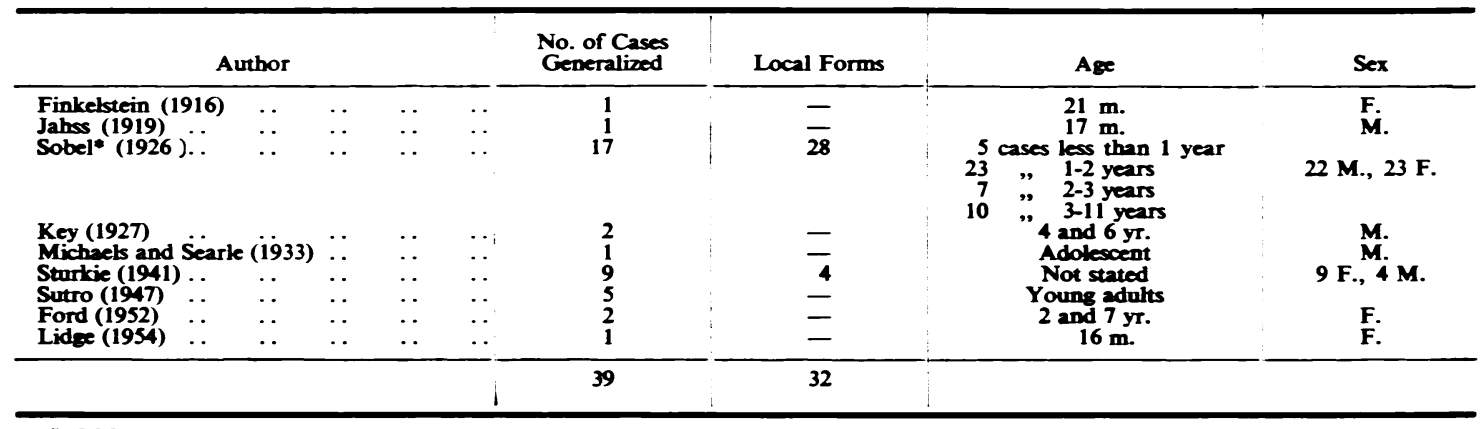

* Of Sobel's 45 cases, 17 appear to be generalined and severe, and would seem to be comparable to the case that we are describing. His other cases were described as moderate or mild, but he gives no criteria for the distinction.

were males and six females. The mother, incidentally, had hypermobility limited to the fingers and thumb only, thus a localized and a generalized form of the condition can occur in the same family. In the case reported here, it is noted that a first cousin is reported to have hypermobile joints.

Clearly the transmission of the hereditary character is irregular and there is considerable variation in expression. Its transmission cannot be explained in terms of Mendelian recessive inheritance.

Of 71 cases of both the generalized and localized forms in which the sex is mentioned, 35 were male and 36 female. These cases are summarized in Table 1. Adults as well as infants are included.

Prognosis and Complications.-The prognosis is excellent, the condition improving as the child grows older. There may be a tendency to constipation because of atonic abdominal musculature, but this was not so in our case. Sutro (1947) described recurrent effusions into the joints in five cases. In three cases both knees were involved and in two cases the ankles.

Hypotonia with laxity of the ligaments may occur in many disorders, and the following classification is offered from the point of view of differential diagnosis.

(1) Primary hypotonia

Congenital laxity of the ligaments.

1. Generalized type.

2. Localized type.

(2) Secondary hypotonia

1. Disorders of the central nervous system.

(a) Cortical degenerations, maldevelopments, and matformations.

(b) Amaurotic family idiocy.

(c) Congenital atonic diplegia (hypotonic cerebral palsy). (d) Injury to the spinal cord.

2. Disorders of the anterior horn cell.

(a) Amyotonia congenita (Oppenheim's disease).

(b) Werdnig-Hoffman disease.

(c) Acute ascending polyneuritis.
3. Atonic type of chorea.

4. Sedative drues.

(3) Muscle dystrophies and atrophies

(4) Endocrine disorders such as hypothyroidism

(5) Nutritional disorders

1. Chronic wasting disorders.

(a) Infections.

(b) Malignancy.

(c) Chronic undernutrition from any cause.

(6) Mongolism

(d) Rickets.

(7) Ellers-Dankos syndrome

(8) Brittle bone disease with blue sclera

Congenital laxity of the ligaments is easily differentiated if the following points are remembered:

(1) There may be a family history of the disorder. (2) The intelligence is unimpaired. (3) Muscle power is normal though the tone is poor. (4) There is hypermobility of the joints which varies in degree and may be localized to one or more joints. (5) The refiexes are normal. (6) Electrical studies are normal. (7) Soft tissue radiographs of the limbs show a normal muscle mass.

\section{Summary}

A case of congenital laxity of the ligaments with hypotonia is presented and the literature is briefly reviewed.

The diagnostic features are emphasized.

I wish to thank Dr. A. Doyne Bell for his help and interest in this case and for permitting me to use the photograph of the child with Werdnig-Hoffman disease. I also wish to thank Mr. W. Smith, Photographic Department, King's College Hospital, for his assistance.

\section{Rererences}

Finkekstein, H. (1916). N.Y. med. J., 104, 942.

Ford, R. (1952). Diseases of the Nervous System in Infancy, Childhood and Adolescence, 3rd ed., p. 320. Oxford.

Jahss, S. A. (1919). N.Y. med. J., 199, 638.

Key, J. A. (1927). J. Amer. med. Ass., 88, 1710.

Lidge, R. T. (1954). J. Pediat., 45, 474.

Michacks, J. J. and Searie, O. M. (1933). J. merv. ment. Dis., 77, 246.

Sobel, J. (1926). Med. J. Rec., 124, 225.

Sturkie, P. D. (1941). J. Hered., 32, 232.

Sutro, C. J. (1947). Surgery, 21, 67. 